\title{
Two-stage treatment (Mukai's method) with hybrid 2-port HALS (Mukai's operation) for complete bowel obstruction by left colon cancer or rectal cancer
}

\author{
MASAYA MUKAI $^{1}$, YASUTOMO SEKIDO ${ }^{2}$, TATSUHIKO HOSHIKAWA $^{1}$, NAOKI YAZAWA ${ }^{1}$, \\ HIROSHI FUKUMITSU ${ }^{1}$, KAZUTAKE OKADA ${ }^{1}$, TAKAYUKI TAJIMA ${ }^{1}$, \\ MASATO NAKAMURA $^{3}$ and KYOUJI OGOSHI ${ }^{4}$
}

Departments of ${ }^{1}$ Surgery and ${ }^{2}$ Pathology, Tokai University Hachioji Hospital, Ishikawa-cho 1838, Hachioji, Tokyo 192-0032; Departments of ${ }^{3}$ Pathology and ${ }^{4}$ Surgery, Bohseidai, Isehara, Kanagawa 259-1193, Japan

Received February 5, 2010; Accepted April 6, 2010

DOI: $10.3892 /$ or_00000824

\begin{abstract}
During the initial emergency operation, a temporary loop colostomy (TLC) was constructed at the oral border of the region for subsequent radical resection. Then, radical surgery was performed by hybrid 2-port HALS (Mukai's operation) using the TLC as the hand access site. Left hemicolectomy was done in three patients, sigmoidectomy in two cases, low anterior resection in one case, and Hartmann's operation in one case. Radical surgery was not done in one patient with multiple distant metastases. The mean operating time was $3 \mathrm{~h}$ and $7 \mathrm{~min}$ (ranging from $1 \mathrm{~h}$ and $55 \mathrm{~min}$ to $3 \mathrm{~h}$ and $47 \mathrm{~min})$, the mean blood loss was $146.4 \mathrm{ml}(7-354 \mathrm{ml})$, the mean duration from TLC to HALS was 11.3 days (8-16 days), and the mean hospital stay after HALS was 13.9 days (9-20 days). Mild wound infection occurred postoperatively in $2 / 7$ patients and ileus occurred in one patient. However, there was no anastomotic leakage/stricture or conversion to conventional laparotomy. These results suggest that 2-stage treatment (Mukai's method with Mukai's operation) is also applicable to large obstructing left colon or rectal cancers. This method is safe, less invasive, and achieves excellent results, including a good cosmetic outcome.
\end{abstract}

Correspondence to: Dr Masaya Mukai, Department of Surgery, Tokai University Hachioji Hospital, Ishikawa-cho 1838, Hachioji, Tokyo 192-0032, Japan

E-mail: mukai.masaya@hachioji-hosp.tokai.ac.jp

Abbreviations: LACS, laparoscopy-assisted colorectal surgery; HALS, hand-assisted laparoscopic surgery; TLC, temporary loop colostomy

Key words: large bowel obstruction, left colon cancer, rectal cancer, 2-stage treatment, laparoscopy-assisted colorectal surgery, handassisted laparoscopic surgery, temporary loop colostomy

\section{Introduction}

In recent years, minimally invasive laparoscopy-assisted colorectal surgery (LACS) has become common, and its indications have expanded considerably from additional resection for early stage I colorectal cancer to radical curative resection of advanced stage II/III cancer and palliative surgery for stage IV patients (1-5). Unlike Western countries, where hand-assisted laparoscopic surgery (HALS) and hybrid-HALS combined with surgery under direct vision are popular (6-9), pure LACS performed via 5-6 ports together with a small incision of $35-45 \mathrm{~mm}$ has become the mainstream procedure in Japan $(10,11)$. However, the use of pure LACS is limited in patients with complete bowel obstruction due to left colon cancer, which is an oncologic emergency, and those who need partial resection of tumors infiltrating other organs such as the bladder. In addition, it has been pointed out that the operating time is relatively long $(5,12-14)$. In order to solve these problems, we devised hybrid 2-port HALS (Mukai's operation), which involves HALS using a small incision of 45-55 $\mathrm{mm}$ as the hand access site, combined with manipulation under direct vision (15). The features of this surgical procedure include the following: i) full grasping manipulation, as well as palpation, can be done with the left hand, enabling protective and smooth handling of even large and heavy tumors; ii) there is little difficulty for anesthesiologists and operating room staff since the procedure is an extension of conventional laparotomy and takes less time than pure LACS and; iii) it is less demanding for surgeons and less time is required to master the procedure, a supervised surgeon who only has experience of standard laparotomy is able to perform it without difficulty. Due to such advantages, hybrid 2-port HALS is applicable to all colorectal resection procedures $(5,13-15)$.

On the other hand, emergency surgery is required in patients with colorectal cancer and complete bowel obstruction. The surgical procedures employed can be classified into those involving resection of the primary tumor and procedures that only relieve obstruction without removing the primary lesion (16-18). When radical resection of the primary tumor 


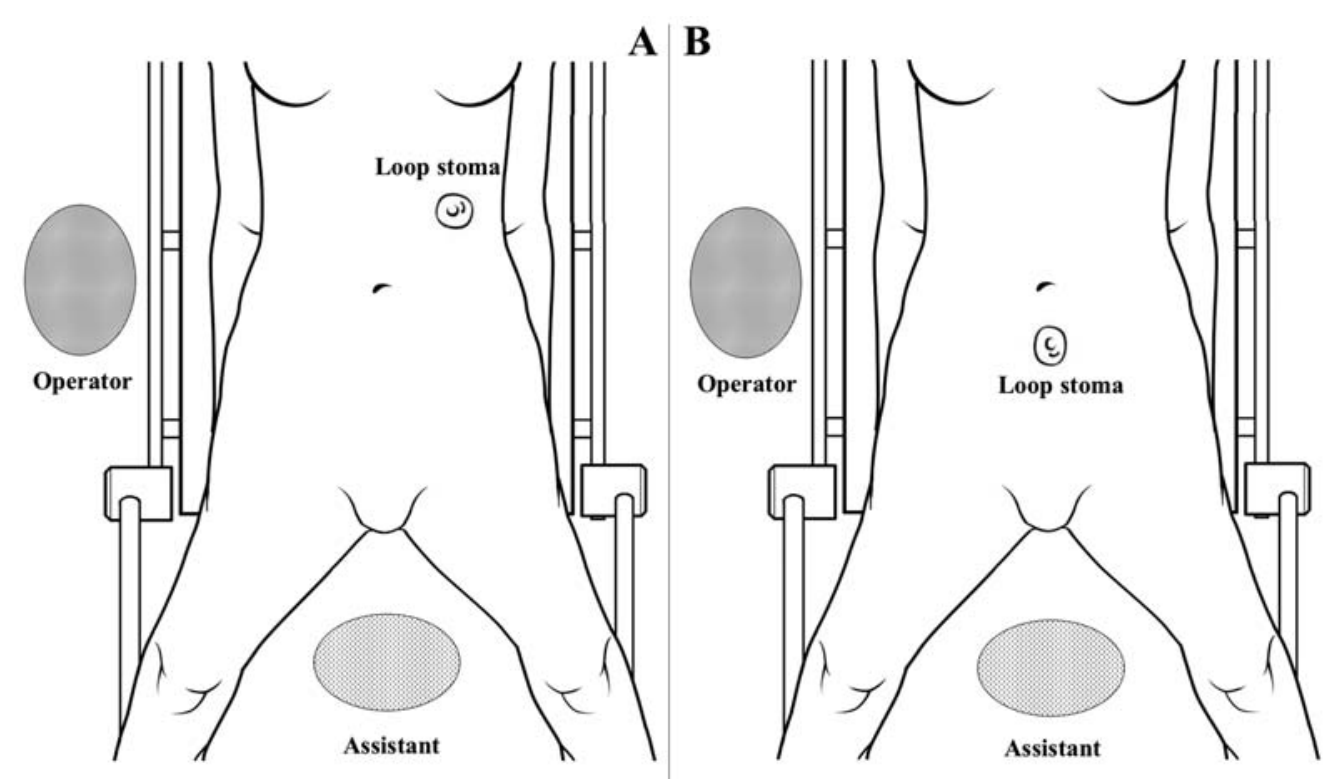

Figure 1. Illustrations of patients in the lithotomy position, and overall view of the abdomen, including a loop colostomy located in the left upper abdomen on the left transverse colon (A) and a loop colostomy located in the lower midline on the mid-sigmoid colon (B).

is followed by anastomosis/reconstruction with construction of a covering colostomy in the right transverse colon, investigation of other tumors and diseases cannot be performed. In addition, the long operating time and highly invasive surgery make it a risky emergency operation (19-21). In Hartmann's operation, anastomosis of the resected stumps is not done and an endocolostomy is constructed on the oral stump following resection of the primary tumor. Because there is a delay until re-anastomosis is performed, there are relatively many cases of difficulty with the anastomotic procedure due to severe pelvic adhesions $(22,23)$. Among the surgical procedures that only relieve obstruction without resecting the primary tumor, construction of a standard temporary loop colostomy on the right transverse colon is a simple and safe method, and it is possible to perform the subsequent elective operation after sufficient evaluation of concomitant diseases and risk factors $(12,17)$. Several reports have suggested that tube cecostomy and loop ileostomy are superior to right transverse colostomy (24-27). At our department, we consider that only construction of a temporary loop colostomy (TLC) should be done, by which safe and secure decompression can be achieved in a short time with little surgical invasion, as the initial emergency operation for patients who have left colon cancer and complete bowel obstruction (12). Regarding the site of the stoma used for decompression, we recommend the bowel on the oral side of the tumor within the range of subsequent second-stage radical resection instead of the standard sites in the right transverse colon and ileum. As a result, we have found that dilatation of the bowel is relieved within approximately 57 days after emergency surgery, and we are able to perform the subsequent elective operation safely following sufficient improvement of the general condition and assessment of risk factors by various examinations (12).

So far, there have been no detailed reports about patients with complete bowel obstruction due to left colon cancer or rectal cancer who underwent construction of a TLC alone as the initial emergency procedure, followed by radical curative resection including the TLC site by hybrid 2-port HALS for 2-stage treatment (Mukai's method with Mukai's operation) using the TLC closure site for hand access after the patient's general condition had improved. Therefore, we studied patients with complete bowel obstruction by left colon cancer or rectal cancer who underwent initial construction of a TLC and subsequent radical resection by hybrid 2-port HALS.

\section{Patients and methods}

During the period of two years and five months from August 2007 to December 2009, we first performed an emergency Gastrograffin enema in patients who were suspected to have complete bowel obstruction due to left colon cancer or rectal cancer and thus confirmed the site of obstruction. Then we performed emergency surgery to construct a TLC on the oral edge of the tumor within the range of subsequent radical resection. We constructed the TLC on the left transverse colon in patients with lesions such as those adjacent to the left transverse colon at the splenic flexure, the descending colon, and the proximal sigmoid colon (Fig. 1A) (5). A site on the mid-sigmoid colon ( $\mathrm{S}$. colon top) was selected for lesions of the distal sigmoid colon and the rectum (Fig. 1B) (13). After ileus was improved, we assessed the bowel to the oral side of the primary tumor and performed sufficient examinations, including evaluation of concomitant diseases and risk factors. Then a radical operation was scheduled for 7-10 days after TLC construction. The second-stage elective operation was done by laparoscopy-assisted surgery (hybrid 2-port HALS; Mukai's operation), using the TLC closure site for hand access (Fig. 2) $(5,13,15)$.

In these patients, we analyzed the site of complete obstruction, the site of TLC, the surgical procedures performed by Hybrid 2-port HALS, and the pathological tumor stage. Furthermore, we calculated the mean operating time 


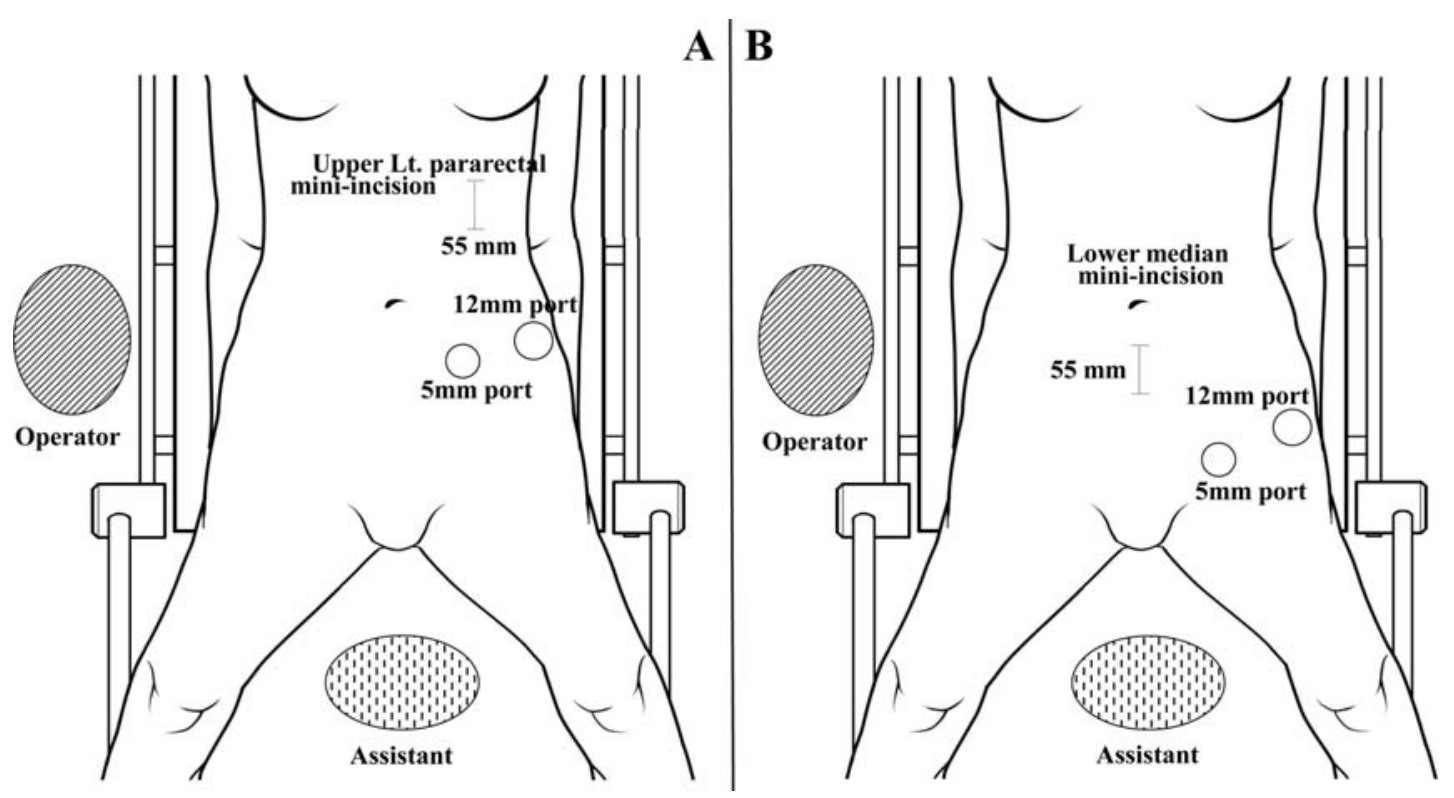

Figure 2. Illustrations of patients in the lithotomy position and an overall view of the abdomen, including a left upper pararectal mini-incision with 2 ports for left hemicolectomy (A); and a lower median mini-incision with 2 ports for sigmoidectomy, low anterior resection and Hartmann's operation (B).

Table I. Eight patients with complete bowel obstruction due to left colon cancer or rectal cancer.

No. of cases

Obstruction site
Left transverse colon
Descending colon
Sigmoid colon
Rectum

Stoma site (temporary loop colostomy)

Left transverse colostomy

Mid-sigmoid colostomy

Hybrid 2-port HALS (Mukai's operation)

Left hemicolectomy D2, 3

Sigmoidectomy D2, 3

Low anterior resection D2

1

Palliative Hartmann's operation

1

Inoperable (distant metastases)

1

Total 8 cases (stage II, 2 cases; stage III, 3 cases; stage IV, 3 cases).

for hybrid 2-port HALS including TLC closure, the mean blood loss, the mean duration from TLC construction to HALS, and the mean hospital stay after HALS. In addition, we analyzed postoperative complications such as wound infection and ileus.

\section{Results}

We have experienced a total of 8 patients with complete bowel obstruction due to left colon cancer or rectal cancer.
Two patients had stage II disease, three had stage III disease, and the remaining three patients were in stage IV (Table I). The site of obstruction was the left transverse colon in one patient, the descending colon in two patients, the sigmoid colon in two patients, and the rectum in three patients (Table I). The TLC was constructed on the left side of the transverse colon in four cases and on the sigmoid colon in four cases (Table I). The surgical procedures performed by hybrid 2port HALS (Mukai's operation) were left hemicolectomy in three patients, sigmoidectomy in two patients, low anterior resection in one patient, and Hartmann's operation in one patient. One patient did not undergo subsequent surgery due to multiple distant metastases (Table I).

Hybrid 2-port HALS (Mukai's operation) was performed in a total of 7 patients. The mean operating time was $3 \mathrm{~h}$ and $7 \mathrm{~min}$ (ranging from $1 \mathrm{~h}$ and $55 \mathrm{~min}$ to $3 \mathrm{~h}$ and $47 \mathrm{~min}$ ), the mean blood loss was $146.4 \mathrm{ml}(7-354 \mathrm{ml})$, the mean duration from construction of the TLC to performance of HALS was 11.3 days (8-16 days), and the mean hospital stay after HALS was 13.9 days (9-20 days) (Table II). Postoperative complications included mild wound infection in $2 / 7$ patients (28.6\%) (Fig. 3) and ileus in one patient (14.3\%). However, anastomotic leakage, anastomotic stricture, and conversion to standard open laparotomy did not occur (Table II).

\section{Discussion}

Emergency surgery is performed in patients with colorectal cancer accompanied by complete bowel obstruction. The larger diameter of the right colon compared with that of the left colon makes it less likely for right colonic lesions to cause complete obstruction. Even if a state of subileus has developed, it is possible to achieve decompression in some cases via a long tube and thus avoid emergency surgery. Also, when emergency surgery is done with no bowel preparation, few surgeons hesitate to perform one-stage resection of the primary tumor and anastomosis since small bowel 
Table II. Results and complications of 2-stage treatment (Mukai's method) by hybrid 2-port HALS (Mukai's operation).

Mean operating time

Mean blood loss

Mean duration to HALS

Complications

Wound infection

Postoperative ileus

Leakage

Anastomotic stricture

Conversion to standard operation
3 h 7 min (min., 1 h 55 min; max., 3 h 47 min)

$146.4 \mathrm{ml}$ (min., $7 \mathrm{ml}$; $\max ., 354 \mathrm{ml}$ )

11.3 days (min., 8 days; max., 16 days)

$2 / 7$ cases $(28.6 \%$, mild $)$

$1 / 7$ cases $(14.3 \%$, conservative therapy)

$0 / 7$ cases $(0.0 \%)$

$0 / 7$ cases $(0.0 \%)$

$0 / 7$ cases $(0.0 \%)$

Total 7 cases, Hybrid 2-port HALS with TLC closure. TLC, temporary loop colostomy.

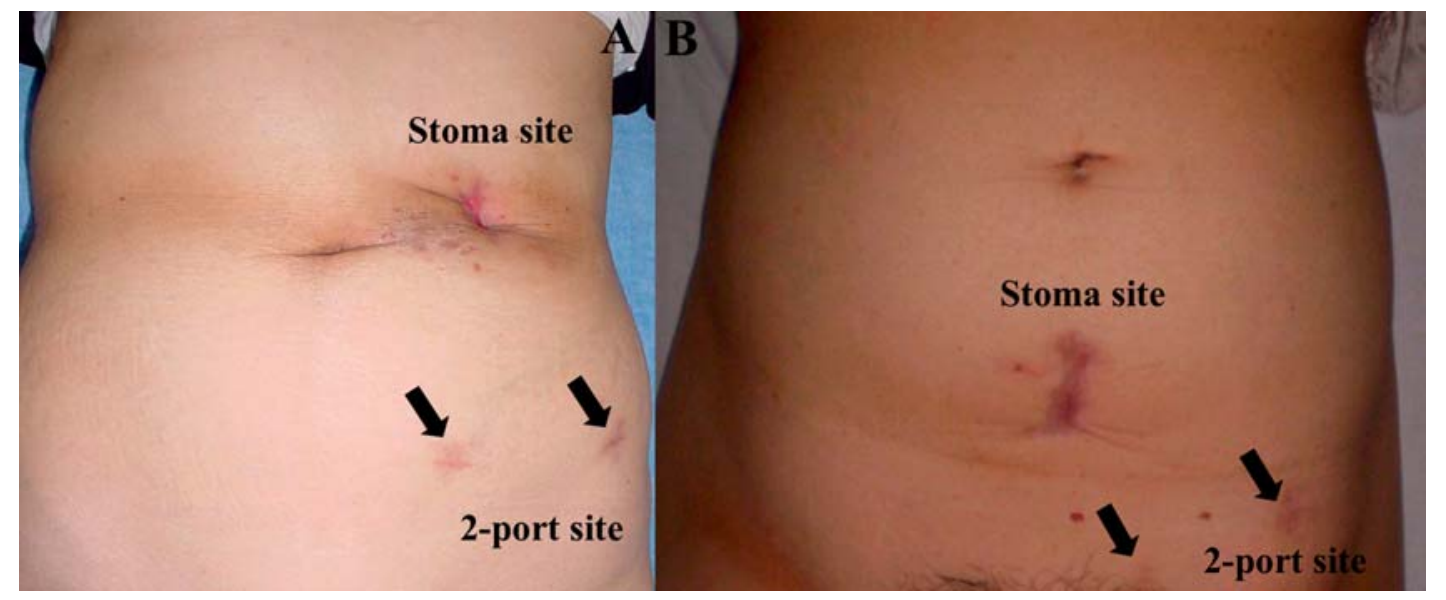

Figure 3. The abdominal wounds at 3-6 months after hybrid 2-port HALS, including a left upper pararectal mini-incision (A) and a lower median miniincision (B).

contents rather than feces is in contact with the anastomosis. Although the frequency was not high in the present study (34 months/case), cases of complete obstruction by left colon cancer, particularly in the descending colon or proximal sigmoid colon where transanal drainage is difficult, are considered to be an oncologic emergency $(5,12,13)$. Because radical resection of the cancer also includes lymph node dissection, construction of a temporary loop ileostomy or temporary loop colostomy on the right transverse colon is performed in most cases after radical resection of the primary tumor with large bowel anastomosis (24-27). There are primary anastomotic procedures involving extensive intraoperative lavage from the oral side of the bowel to avoid the construction of a temporary stoma, but these procedures require a relatively long operating time and also may be excessively invasive for emergency surgery in elderly patients $(28,29)$. Also, there have been reports of serious complications of such procedures, including intraoperative rupture of dilated bowel, DIC, wound infection and dehiscence, anastomotic leakage, and pelvic floor peritonitis $(30,31)$. We have already reported decompression by constructing a loop colostomy at the oral edge of the tumor within the range of subsequent resection by radical surgery, and after the general condition of the patient has improved and sufficient evaluation of concomitant diseases and risk factors has been performed, radical excision of the tumor and combined resection of the colostomy were done by elective standard median laparotomy in patients with complete bowel obstruction due to left colon cancer (12). Although it involved a 2-stage operation with 2 wounds due to stoma closure site, the advantages included the following: there was only one anastomotic site and no limitation on the extent of radical curative resection, and there were few complications caused by stoma closure such as anastomotic leakage or stenosis. Also, it was applicable to patients who need extensive resection because of multiple colorectal cancers (12). In the present study, we applied the hybrid 2-port HALS method for colorectal cancer patients with ileus using small incisions and employed the stoma closure site for hand access $(5,13,15)$. When we introduced this method, wound infection at the hand access site was a concern, but mild wound infection only occurred in 2/7 cases. This may have been due to the extremely short period with the stoma, which was 11.3 days in our series, but detailed analysis will be needed in the future. 
In Japan, pure LACS has become widely performed as minimally invasive surgery. However, there are problems such as difficulty in obtaining a sufficient number of surgeons who have mastered LACS in order to shorten the operating time and reduce the pressure on anesthesiologists and operating room staff. In fact, LACS has not become the gold standard operation even at comparatively large general hospitals and some institutions have even abandoned routine performance of LACS. During pure LACS, operative manipulation is mainly performed with four sets of forceps by the operator and the first assistant, so at least two skilled surgeons are needed including one who is skilled in smooth handling of the camera. Unlike conventional laparotomy, the following problems arise with LACS: i) lack of palpation/tactile sentation, ii) limited applicability in patients with large and heavy tumors, iii) difficulty in grasping the overall features of the surgical field in relation to the extent of resection, iv) dependence on visual perception that leads to a longer operating time, and v) difficulty in mastering the relevant skills and the need to obtain technical certification in Japan. On the other hand, hybrid 2-port HALS is different from pure LACS. In addition to anastomosis, procedures such as the pelvic floor plasty for complete closure of the mesocolon/ mesorectum after colorectal resection, ligation/dissection of vessels such as the colic arteries and veins, and lymph node dissection are comparatively simple to perform under direct vision and/or camera guided vision. It is also thought that hybrid 2-port HALS is safely applicable to patients with ileus and those who need combined resection of tumors infiltrating other organs (5,12-15). Hybrid 2-port HALS is performed as the routine elective operation at our department, and if the left hand can reach the posterior side of the tumor, there are no major differences of dissection layer compared with manipulation by standard laparotomy. In addition, manipulations that used to be performed almost blindly during standard laparotomy at sites that were hard to observe, such as the pelvic floor, the inferior wall of the bladder, and the posterior wall of the prostate, can be performed safely while being viewed on the monitor with magnification. Therefore, hybrid 2-port HALS is thought to be useful for almost all patients. Hybrid 2-port HALS is generally indicated for patients with i) the ability to tolerate general anesthesia for approximately $2 \mathrm{~h}$, ii) no major concomitant diseases such as cardiac/ pulmonary disease, and iii) preferably a P.S. of 0-1 and an age younger than 75 years. In addition, the patient should be free from detectable lateral lymph node metastasis and the tumor should not fill the pelvic cavity in patients with advanced rectal cancer $(15,32)$. A transverse incision of $55 \mathrm{~mm}$ at the superior border of the pubis is regarded as standard for patients with advanced lower rectal cancer. In addition, dissection of metastatic lateral lymph nodes is possible under direct vision by making a small incision, such as a lower median incision or lower pararectal incision that can also be used for hand access site near the site of the metastatic nodes (32). More patients, including those with advanced rectal cancer, need to be studied in the future, and a detailed comparison of data such as recurrence rates including local recurrence in the pelvic cavity and distant metastasis, is needed to fully assess the value of 2-stage treatment (Mukai's method) by hybrid 2port HALS (Mukai's operation).

\section{Acknowledgements}

This study was supported by grants from the Occult Neoplastic Cells Research and Study Group (No. 2009-5007; Tokai University Hachioji Hospital, Tokyo, Japan) and the Research and Study Program of Tokai University Educational System General Research Organization (No. 2007-04; Tokai University School of Medicine, Kanagawa, Japan).

\section{References}

1. Mukai M, Tokunaga N, Ishida H, Makuuchi H, Tajima T and Mitomi T: Clinical experiences with laparoscopic colectomy. Dig Endosc 9: 11-15, 1997.

2. Mukai M, Okamoto Y, Oida Y, Mukoyama S, Ito I, Nakasaki H, Kawai K, Sato S and Makuuchi H: Endoscopic mucosal resection for superficially spreading colonic neoplasms larger than $5 \mathrm{~cm}$ in the right colon after dilute sodium hyaluronate injection: report of two cases. Endoscopy 35: 973-975, 2003.

3. Sugihara K and Makuuchi M: Knacks and Pit falls: Surgery of the Colon, Rectum and Anus. 2nd edition. Bunkoudou, Co., Ltd., Tokyo, 2004.

4. Treatment guide line of the large bowel cancer in Japan. Japanese Society for Cancer of the Colon and Rectum. Tokyo, 2009.

5. Mukai M, Tanaka A, Tajima T, Yamagiwa T, Okada K, Fukasawa M, Sato K, Oida Y, Nakamura M and Makuuchi H: Two-port hand-assisted laparoscopic surgery (HALS) in 2-stage treatment for complete obstruction by left colon cancer: a case report. Oncol Rep 19: 875-879, 2008.

6. Romanelli JR, Kelly JJ and Litwin DE: Hand-assisted laparoscopic surgery in United States: an overview. Semin Laparosc Surg 8: 96-103, 2001.

7. Vithiananthan S, Cooper Z, Betten K, Stapleton GS, Carter J, Huang EH and Whelan RL: Hybrid laparoscopic flexure takedown and open procedure for rectal resection is associated with significantly shorter length of stay than equivalent open resection. Dis Colon Rectum 44: 927-935, 2001.

8. Nakajima K, Lee SW, Cocilovo C, Foglia C, Kim K, Sonoda T and Milson JW: Hand-assisted laparoscopic colorectal surgery using GelPort. Surg Endosc 18: 102-105, 2004.

9. Nakajima K, Lee SW, Cocilovo C, Foglia C, Sonoda T and Milson JW: Laparoscopic total colectomy: hand-assisted vs. standard technique. Surg Endosc 18: 582-586, 2004.

10. Jacobs M, Verdja JC and Goldstein HS: Minimally invasive colon resection (laparoscopic colectomy). Surg Laparosc Endosc 1: 144-150, 1991.

11. The Clinical Outcomes of Surgical Therapy Study Group: A comparison of laparoscopically assisted and open colectomy for colon cancer. N Engl J Med 350: 2050-2059, 2004.

12. Mukai M, Himeno S, Mukoyama S, Tajima T, Saito Y, Ito I, Nakasaki H, Sato S and Makuuchi H: Is the temporally loop colostomy in the right transverse colon appropriate for complete obstruction by colorectal cancer? Oncol Rep 10: 693-698, 2003.

13. Mukai M, Kishima K, Iizuka S, Fukumitsu H, Fukasawa M, Yazawa N, Tajima T, Nakamura $\mathrm{M}$ and Makuuchi H: Hybrid 2-port HALS for 2-stage treatment of complete obstruction by distal sigmoid colon cancer: A case report. Oncol Rep 21: 1203-1208, 2009.

14. Mukai M, Kishima K, Iizuka S, Fukumitsu H, Fukasawa M, Yazawa N, Tajima T, Nakamura M and Makuuchi H: Curative resection by hybrid 2-port HALS in a patient with advanced cecal cancer invading the urinary bladder: A case report. Oncol Rep 21: 1385-1389, 2009.

15. Mukai M, Kishima K, Tajima T, Hoshikawa T, Yazawa N, Fukumitsu H, Okada K, Ogoshi K and Makuuchi H: Efficacy of hybrid 2-port hand-assisted laparoscopic surgery (Mukai's operation) in patients with colorectal cancer. Oncol Rep 22: 893-899, 2009.

16. Hoffmann J and Jensen HE: Tube cecostomy and staged resection for obstructing carcinoma of the left colon. Dis Colon Rectum 27: 24-32, 1984 .

17. Mackenzie S, Thompson SR and Baker LW: Management options in malignant obstruction of the left colon. Surg Gynecol Obstet 74: 337-344, 1992. 
18. Sjödahl R, Franzen T and Nyström PO: Primary versus staged resection for acute obstructing colorectal carcinoma. Br J Surg 79: 685-688, 1992.

19. Stothert JC Jr, Brubacher L and Simonowitz DA: Complications of emergency stoma formation. Arch Surg 120: 1145-1147, 1985.

20. Pakkastie TE, Ovaska JT, Pekkara ES, Luukkonen PE and Jarvinen HJ: A randomized study of colostomies in low rectal anastomoses. Eur J Sur 163: 929-933, 1997.

21. Dehni N, Schlegel RD, Cunningham C, Guiguet M, Tiret E and Pare R: Influence of a defunctioning stoma on leakage rates after low colorectal anastomosis and colonic J pouch-anal anastomosis. Br J Sur 85: 1114-1117, 1998.

22. Schein M and Decker G: The Hartmann procedure: extended indications in severe intra-abdominal infection. Dis Colon Rectum 31: 126-129, 1987.

23. Keck JM, Collopy BT, Ryan PJ, Fink R, Mackay JR and Woods RJ: Reversal of Hartmann's procedure: effect of timing and technique on ease and safety. Dis Colon Rectum 37: 243-248, 1994.

24. Gooszen AW, Geelkerken RH, Hermans J, Lagaay MB and Gooszen HG: Temporary decompression after colorectal surgery: randomized comparison of loop ileostomy and loop colostomy. Br J Surg 85: 76-79, 1998.

25. Tschmelitsch J, Wykypiel H, Prommegger R and Bodner E: Colostomy vs tube cecostomy for protection of low anastomosis in rectal cancer. Arch Surg 134: 1385-1388, 1999.
26. Rullier E, Le Toux N, Laurent C, Garrelon J-L, Parneix M and Saric J: Loop ileostomy versus loop colostomy for defunctioning low anastomoses during rectal cancer surgery. World J Surg 25: 274-278, 2001.

27. Edwards DP, Leppington-Clarke A, Sexton R, Heald RJ and Moran BJ: Stoma-related complications are more frequent after transverse colostomy than loop ileostomy: a prospective randomized clinical trial. Br J Surg 88: 360-363, 2001.

28. Thomson WH and Carter SS: On-table lavage to achieve safe restorative rectal and emergency left colonic resection without covering colostomy. Br J Surg 73: 61-63, 1986.

29. Pollock AV, Playforth MJ and Evans M: Peroperative lavage of the obstructed left colon to allow safe primary anastomosis. Dis Colon Rectum 30: 171-173, 1987.

30. Karanjia ND, Corder AP, Holdsworth PJ and Heald RJ: Risk of peritonitis and fatal septicaemia and the need to defunction the low anastomosis. Br J Sur 78: 196-198, 1991.

31. Porter JA, Salvati EP, Rubin RJ and Eisentat TE: Complications of colostomies. Dis Colon Rectum 32: 299-303, 1989.

32. Mukai M, Fukasawa M, Kishima K, Iizuka S, Fukumitsu H, Yazawa N, Tajima T, Nakamura M and Makuuchi H: Transanal reinforcing sutures after double stapling for low rectal cancer: Report of two cases. Oncol Rep 21: 335-339, 2009. 